Paper:

\title{
Sliding Mode Robot Control with Friction and Payload Estimation
}

\author{
Lớrinc Márton*, and Béla Lantos** \\ *Dept. of Electrical Engineering, Sapientia Hungarian University of Transylvania \\ 540053 Tg. Mures, P-ta. Trandafirilor 61, Romania \\ E-mail: martonl@ms.sapientia.ro \\ ** Dept. of Control Engineering and Information Technology, Budapest University of Technology and Economics \\ H-1117 Budapest, Magyar Tudósok körútja 2, Hungary \\ E-mail: lantos@iit.bme.hu \\ [Received May 5, 2004; accepted June 16, 2004]
}

\begin{abstract}
The paper deals with robust motion control of robotic systems with unknown friction parameters and payload mass. The parameters of the robot arm were considered known with a given precision. To solve the control of the robot with unknown payload mass and friction parameters, sliding mode control algorithm was proposed combined with robust parameter adaptation techniques. Using Lyapunov method it was shown that the resulting controller achieves a guaranteed final tracking accuracy. Simulation results are presented to illustrate the effectiveness and achievable control performance of the proposed scheme.
\end{abstract}

Keywords: robot control, sliding control, adaptive compensation, Lyapunov stability

\section{Introduction}

\subsection{Motivation}

Nowadays the robotic applications require increased transient performances and path tracking proprieties. To achieve these requirements the introduction of the mathematical model in the control algorithm is necessary. There are analytical methods to determine the exact mathematical model of a robot, for example using the EulerLagrange method [1-3]. The parameters of the model (masses, inertias, length of the arms) are often catalog data which are given by the manufacturer of the robotic system $[4,5]$. However especially in the case of the inertias the value of the parameters cannot be determined exactly, they are known with a given precision. This uncertainty should also be taken into consideration in the control algorithm.

The friction phenomena which should be considered in every mechanical system can be described with models whose parameters are time varying, depending external factors. These parameters cannot be determined a-priori. At the other hand there is a tendency in robotic industry to build robots with lightweight arms to avoid unnecessary energy consumption. For this reason in the robot model the mass of the payload cannot be neglected related to the masses of the arms. The mass of the payload in many application is unknown, varies according to the specific task of the robot. These considerations suggest that the friction parameters and the mass of the payload should be estimated on-line. If only the friction forces and the payload are unknown in the model it is unnecessary to use adaptive control algorithms which estimate all the parameters of the robot.

\subsection{Previous Works}

In the past years the robust adaptive control methods [6] became a wide spread method to control not only the linear but also the nonlinear systems with unknown parameters. Robust adaptive control of robotic manipulators has also been intensively studied in the control community.

Early results can be found in the work of Slotine and Li [7] who proposed the introduction of a simple switching term in the adaptive robot control algorithm to handle modelling errors.

The introduction of a neural network in the adaptive control law to handle uncertainties due to payload uncertainty was proposed by Leahy et al. [8]

A decentralized robust adaptive control algorithm for trajectory tracking was proposed by $\mathrm{Fu}$ [9]. The controller can be implemented in a decentralized way, i.e., a subcontroller is independently and locally equipped for each joint servo loop.

A robustified adaptation law (switching $\sigma$ modification) combined with sliding control was proposed for single arm robotic systems by Chen and Papavassilopoulos [10] to increase the robustness of the control system and to improve the tracking error performance.

Adaptive disturbance attenuating controller was proposed by Tomei for robotic manipulators without [11] and with [12] friction compensation. It was considered that all the disturbances are bounded and if the disturbance vanishes the controller achieves asymptotic tracking.

Artega [13] proposes an adaptive scheme designed in conjunction with a linear velocity observer. Boundedness of the estimated parameters, tracking error and state estimation are guaranteed. 
In many works it is considered that the model of the controlled robot is completely unknown. In this case in the feedforward term of the controller neural or fuzzy models [14] are introduced to compensate the effect of nonlinearities and model uncertainties. The parameters of these models are tuned on-line.

Lewis et al. [15] combine the neural control technique with linear optimal control theory to control rigid manipulators with completely unknown models.

An adaptive neural-network based tracking control with guaranteed $H^{\infty}$ performances was proposed for robotic systems with plant uncertainties and external disturbances by Chang and Cheng [16].

Barambones and Etxebarria [17] proposes a controller for generic manipulator with unknown parameters and sliding-mode control which robustifies the design and compensates the neural approximation errors.

Many works deal with the control of square nonlinear Multi Input Multi Output (MIMO) systems with poorly understood dynamics. The developed controllers could also be applied for robot control. Direct and indirect fuzzy adaptive control schemes are proposed by Ordonez and Passino [18]. The robustness of the adaptation is increased with parameter projection algorithms [19, 20]

In the work of Chang [21] fuzzy control methods are combined with $H^{\infty}$ control theory to design robust output tracking controller for nonlinear MIMO systems.

Nonlinear robust adaptive control algorithm for MIMO systems with unknown nonlinearities using switching functions and RBF type neural networks was used by $\mathrm{Xu}$ and Ioannou [22].

\subsection{Robot Modelling}

The mathematical model of an open chain, rigid, $n$ degree of freedom robot is given by:

$$
H(\underline{q}) \underline{\ddot{q}}+C(\underline{q}, \underline{\dot{q}}) \underline{\dot{q}}+D(\underline{q})=\underline{\tau}-\underline{h}_{F}(\underline{\dot{q}}) . . .
$$

where $q$ is the joint position vector of the robot and the vector $\frac{\bar{\tau}}{\underline{\tau}}$ represents the control signal realized by current controlled DC servos. The following notations is used: $H(\underline{q})$ is the generalized inertia matrix, $C(\underline{q}, \dot{q})$ shows the effect of the centripetal and Coriolis forces, $D(\underline{q})$ is the gravity effect, $\underline{h}_{F}(\dot{q})$ represents the effect of the friction force which acts on the joints of the robot.

The following properties of the robot model are well known [1]:

1 The matrix $H(\underline{q})$ is positive definite

2 The matrix $\dot{H}(\underline{q})-2 C(\underline{q}, \underline{\dot{q}})$ is skew-symmetric, namely for any $\underline{x} \in R^{n}$ we have:

$$
\underline{x}^{T}(\dot{H}(\underline{q})-2 C(\underline{q}, \underline{\dot{q}})) \underline{x}=0 .
$$

\subsection{Assumptions}

To develop a control law with friction and payload estimation in the presence of modelling errors firstly let us consider the following assumptions:

Assumption Ia (Payload)
Let us assume that the dimensions of the payload can be neglected compared with the dimensions of the robotic arm so its length and inertia is neglected. In this case the terms of the robotic model can be written in the following form:

$$
\begin{aligned}
H(\underline{q})= & \left.H_{R}(\underline{q})+m H_{L}(\underline{q})\right) . \ldots . \\
C(\underline{q}, \underline{\dot{q}}) \dot{q}+D(\underline{q})= & C_{R}(\underline{q}, \underline{\dot{q}}) \dot{\dot{q}}+D_{R}(\underline{q}) \\
& +m\left(C_{L}(\underline{q}, \underline{\dot{q}}) \underline{\dot{q}}+D_{L}(\underline{q})\right) .
\end{aligned} .
$$

where $m$ is the mass of the payload. $H_{R}, C_{R}$ and $D_{R}$ are terms of the robotic arm model without payload. $H_{L}, C_{L}$ and $D_{L}$ are known terms which can be determined from the robot model and do not contain the payload and friction parameters. The analysis of these terms are presented in Appendix A.

\section{Assumption Ib (Payload)}

The mass of the payload is bounded, namely $0 \leq m \leq$ $m_{M}$, where $m_{M}$ is the known upper bound of $m$.

\section{Assumption IIa (Modelling errors)}

The modelling errors for the robotic arm are bounded with known bounds, i.e.

$$
\begin{aligned}
& H_{R}(\underline{q})=H_{R C}(\underline{q})+\Delta H_{R}(\underline{q}) \ldots \ldots . . . . \\
& C_{R}(\underline{q}, \underline{\dot{q}}) \underline{\dot{q}}+D_{R}(\underline{q}) \\
& \quad=C_{R C}(\underline{q}, \underline{\dot{q}}) \underline{\dot{q}}+D_{R C}(\underline{q})+\Delta C_{R}(\underline{q}, \underline{\dot{q}}) \underline{\dot{q}}+\Delta D_{R}(\underline{q}) .
\end{aligned}
$$

where $H_{R C}, C_{R C}$ and $D_{R C}$ are approximately known terms of the robot model that are used in control algorithm and $\Delta H_{R}, \Delta C_{R}, \Delta D_{R}$ is the matrix and the vectors which contains the modelling errors. Each element of these terms are bounded:

$$
\begin{aligned}
& \left|\left(\Delta H_{R}\right)_{i j}\right| \leq\left(\Delta H_{R}\right)_{i j M}, \quad\left|\left(\Delta C_{R}\right)_{i}\right| \leq\left(\Delta C_{R}\right)_{i M} \\
& \left|\left(\Delta D_{R}\right)_{i}\right| \leq\left(\Delta D_{R}\right)_{i M} \text {. . . . . . . . . . . . }
\end{aligned}
$$

where $\left(\Delta H_{R}\right)_{i j M},\left(\Delta C_{R}\right)_{i M},\left(\Delta D_{R}\right)_{i M}$ are known upper bounds.

\section{Assumption IIb (Modelling errors)}

The unmodelled dynamics and external disturbances can be incorporated in a vector $\underline{d}$ which appears as an additive term in the robot model. Each element of this vector is bounded with known bounds:

$$
\left|d_{i}\right| \leq d_{i M} .
$$

\section{Assumption III (Friction Modelling)}

The friction force acts separately on each joint of the robot. It can be written in a linearly parameterized form:

$$
h_{F i}\left(\dot{q}_{i}\right)=\mu\left(\dot{q}_{i}\right) \underline{\theta}_{F P i}^{T} \underline{\xi}_{F P i}\left(\dot{q}_{i}\right)+\left(1-\mu\left(\dot{q}_{i}\right)\right) \underline{\theta}_{F N i}^{T} \underline{\xi}_{F N i}\left(\dot{q}_{i}\right)
$$

where $\mu\left(\dot{q}_{i}\right)=1$ if $\dot{q}_{i} \geq 0$ and 0 otherwise. The vectors $\underline{\theta}_{F i}$ contains the unknown parameters of the friction model and $\underline{\xi}_{F i}\left(\dot{q}_{i}\right)$ are known regressor vectors. This relation means that the friction can be described by two separate linearly parameterized models, one for the positive $(\mathrm{P})$ velocity regime and the other for negative $(\mathrm{N})$ velocity regime. The switching between these two models occurs at the zero velocity. If we incorporate the switching func- 